\title{
Beneficios compartidos y la gobernanza de la extracción de recursos naturales en territorios indígenas: aportes y limitaciones para Latinoamérica
}

\author{
Gonzalo Bustamante-Rivera, ${ }^{*}$ Thibault Martin ${ }^{\dagger * *}$
}

Perfiles Latinoamericanos, 26(52) | 2018

DOI: $10.18504 / \mathrm{pl} 2652-009-2018$

Recibido: 25 de febrero de 2015

Aceptado: 20 de septiembre de 2017

\begin{abstract}
Resumen
Los beneficios compartidos corresponden a una modalidad reciente de gobernanza de la extracción de recursos naturales en territorios indígenas en la que las industrias extractivas comparten directamente con las comunidades afectadas una parte de los beneficios obtenidos en sus operaciones. En el centro del debate se encuentra el tema del rol del Estado ante los proyectos extractivos, el carácter asimétrico de las negociaciones directas entre comunidades e industrias extractivas, sus reales resultados para las comunidades y la naturaleza de las negociaciones para acordar los beneficios compartidos. Se discute su aplicabilidad en el contexto latinoamericano.
\end{abstract}

\begin{abstract}
Benefit sharing is a type of governance of natural resources extraction on indigenous lands where extractive industries share directly with affected communities a part of the benefits of the project. Within the core of these debates we find the role of the State regarding extractive projects, the asymmetry character of direct negotiations between communities and extractive industries, the real outcomes for communities and the nature of these negotiations to agree benefit sharing. Their implications for Latin America are discussed.
\end{abstract}

Palabras clave: gobernanza, pueblos indígenas, recursos naturales-explotación, beneficios compartidos, América Latina.

Keywords: Governance, indigenous peoples, natural resources-exploitation, benefit sharing, Latin America.

* Magister en Desarrollo Local y Regional por la Universidad de La Frontera. Académico, Universidad de La Frontera, Chile | busg03@uqo.ca.

** †Ph.D. en Sociología por la Universidad Laval. Académico, Université du Québec en Outaouais. 


\section{Introducción ${ }^{1}$}

E

las últimas décadas se ha producido un aumento considerable en la extracción de recursos naturales tanto en países desarrollados como en vías de desarrollo (Campbell \& Laforce, 2010; Reed, 2002; Gudynas, 2012). En este contexto, diversos organismos e informes internacionales han identificado a los pueblos indígenas como uno de los grupos de población más afectados (World Commission on Dams, 2000; Anaya, 2011).

Las instituciones financieras internacionales, los gobiernos y las compañías transnacionales han promovido los proyectos extractivos argumentando que estos promueven el desarrollo económico sustentable (Sawyer \& Gómez, 2012). Sin embargo, este argumento es cuestionado por quienes sustentan el debate sobre la "maldición de la abundancia", mismos que destacan la importancia de elaborar mecanismos de gobernanza adecuados para que la abundancia se traduzca efectivamente en desarrollo y bienestar para toda la población (Pendergast et al., 2011; Dietz et al., 2007; Ross, 1999; Salant, 1995; Dunning, 2005; Boschini et al., 2007; Weinthal \& Jones, 2006; Auty, 1998).

En América Latina gran parte de los países han optado por el desarrollo basado en la extracción de recursos naturales, pero no han hecho cambios significativos en la gobernanza relativa a la participación de las comunidades locales afectadas (Bebbington, 2012). Además, el beneficio directo que estos proyectos aportan a las comunidades es, a decir lo menos, incierto. En cambio, en el mundo anglosajón, en las últimas décadas el aumento de la extracción de recursos naturales se ha acompañado de la implementación de nuevas modalidades de gobernanza para estas actividades (Campbell \& Sarrasin, 2012).

En este artículo se analiza una de estas modalidades que el Banco Mundial llama beneficios compartidos (benefit sharing, en inglés) (en adelante, BC). En especial, los que se negocian con comunidades indígenas. Por $\mathrm{BC}$ se entiende toda negociación directa entre comunidades indígenas y gobierno o industrias extractivas, en virtud de lo cual reciben cualquier beneficio resultante de la exploración y explotación de los recursos naturales existentes en sus territorios o cerca de ellos.

En América Latina, los BC se han utilizado de forma poco sistemática y han sido escasamente investigados. Esto contrasta con la abundante literatura del mundo anglosajón, sobre todo acerca de Canadá y Australia, donde los BC

1 Los autores agradecen a los dirigentes de la comunidad de huascoaltinos del pueblo diaguita en Chile por sus comentarios que permitieron precisar algunos contenidos de este artículo. 
se usan en los proyectos mineros, petroleros e hidroeléctricos. Hasta 2012, en Canadá se habían firmado 182 acuerdos de BC.

Para cumplir el objetivo de este artículo se ha realizado una búsqueda y análisis de la literatura científica, la mayor parte correspondiente a la modalidad de "Acuerdos de impactos y beneficios" (en adelante, AIB) en Canadá y Australia y al uso de $\mathrm{BC}$ en casos hidroeléctricos en diversos países. Debido a que los $\mathrm{BC}$ han predominado en la minería y en Canadá, el debate en la materia toma varias de sus particularidades.

Nuestro análisis puede resultar esclarecedor para países latinoamericanos cuyos regímenes mineros se basan, al igual que en Canadá, en el principio de libre entrada (free entry, en inglés) o minería libre (free mining, en inglés). Bajo este principio, los proyectos mineros cobran prioridad por sobre otros usos de las tierras públicas, o que comparten también el que la minería es una fuente de desarrollo económico prioritario para el país.

Asimismo, como los mecanismos de BC son intensivamente utilizados en Canadá por compañías mineras que operan en América Latina, también se usan en proyectos hidroeléctricos que promueve el Banco Mundial; y al entrar en Latinoamérica (aunque no sistemáticamente), se puede hipotetizar que su uso habrá de crecer en los próximos años, lo que hace necesario detectar posibles tensiones y aportes que esos mecanismos pudieran acarrear para la región, justamente, la aportación de este artículo.

\section{La gobernanza de la extracción de recursos naturales en territorios indígenas en América Latina}

En América Latina numerosos estudios coinciden en identificar el magro aporte que los proyectos extractivos significan para el desarrollo local (Bebbington, 2012; Arellano-Yanguas, 2012; Zarsky \& Stanley, 2011), la debilidad estructural del Estado en la gobernanza de las actividades extractivas (Bebbington, 2012; Laforce, 2012a; Casalis \& Trinelli, 2013; Ostau \& Niño, 2012), y en las escasas innovaciones a la gobernanza que los gobiernos posneoliberales han introducido en los años recientes (Bebbington, 2012; Gudynas, 2012).

De acuerdo a Bebbington (2012), las perspectivas teóricas en el estudio sobre la extracción de recursos naturales en América Latina se dividen entre los críticos o "pesimistas" que enfatizan los efectos negativos de los proyectos extractivos en la vida de las comunidades, los "optimistas" que promueven el modelo extractivo, y los "institucionalistas" que se centran en analizar las instituciones que

2 Impact and Benefit Agreements, en inglés; Ententes sur les répercussions et les avantages, en francés.

G. Bustamante-Rivera, T. Martin | Beneficios compartidos y la gobernanza de la extracción de recursos naturales en territorios indígenas: aportes y limitaciones para Latinoamérica | Perfiles Latinoamericanos, 26(52) 
conforman los regímenes de extracción de recursos. Al revisar esta literatura, queda claro que en su mayor parte analiza los regímenes legales e institucionales a nivel nacional o local de la extracción minera o petrolera y en especial los conflictos que producen estas actividades.

En el ámbito específico del uso de mecanismos de gobernanza que aseguren una distribución directa de los ingresos derivados de los proyectos extractivos en las localidades afectadas, se han estudiado escasos ejemplos: en Argentina, el uso de empresas mineras públicas provinciales (Casalis \& Trinelli, 2013); en Perú, el "canon minero" que establece que una parte de los ingresos por royalties se debe distribuir directamente en la localidad donde operan los proyectos extractivos (Arellano-Yanguas, 2012), y en Bolivia, los cambios legales para que las comunidades indígenas se beneficien con un $5 \%$ de los ingresos obtenidos de los impuestos de los proyectos extractivos (Bebbington, 2012).

El otro mecanismo utilizado en América Latina corresponde a los BC, el foco de interés en este artículo. No obstante, si bien encontramos estudios acerca de los BC, solo Laforce (2012a) analiza el uso de estos mecanismos. Por ejemplo, en el informe de Égré (2007) se menciona su aplicación en las centrales hidroeléctricas de Urrá 1 en Colombia y de Itaipú en Brasil y Paraguay, sin embargo, no considera que ambos casos son fuertemente criticados por sus altos impactos negativos sobre las comunidades indígenas afectadas y porque los $\mathrm{BC}$ se implementaron por orden judicial en Urrá 1 y solo después de dos décadas de ejecutado el proyecto en Itaipú. En Chile, la central hidroeléctrica Ralco utilizó algunas formas de BC (compensaciones individuales y financiamiento de programas de desarrollo comunitario), pero Johnston \& GarcíaDowning (2004) describen las fallas tanto de la empresa como del gobierno para negociar los acuerdos. Por su parte, Zarsky \& Stanley (2011) abordan los beneficios directos e indirectos obtenidos por Guatemala y por las comunidades afectadas de los ingresos de la mina Marlin y concluyen que las comunidades reciben solo un $5 \%$ de los ingresos (en gran parte como beneficios indirectos), y el gobierno nacional un $41 \%$ del total. En Bolivia, Riva (2010) describe las negociaciones entre comunidades indígenas y el gobierno para obtener el consentimiento previo, libre e informado (en adelante, CPLI) de las comunidades ante las exploraciones sísmicas, donde se incluyen de forma secundarias mecanismos de BC. En Perú, Arellano-Yanguas (2012) menciona los Programas Mineros de Solidaridad con el Pueblo (que parecen ser modalidades de BC), pero no las describe ni analiza. Solo Laforce (2012a) se ocupa del uso de $\mathrm{BC}$ en casos mineros en Perú y concluye que corresponden a mecanismos positivos, pero que no resuelven los problemas de asimetría de poder. También en Perú, el reciente reglamento de la Ley de Consulta Previa solo menciona los $\mathrm{BC}$ en una de sus "disposiciones complementarias, transi- 
torias y finales", pero no especifica cómo se integra con los contenidos de la consulta previa y no hay estudios sobre su aplicación. Finalmente, en Chile la compañía minera Barrick Gold ha comenzado a utilizar este tipo de mecanismos con las comunidades indígenas, ${ }^{3}$ no obstante, estas negociaciones han resultado altamente conflictivas.

Estos datos muestran que los mecanismos de BC ya se utilizan en América Latina, pero que no han recibido suficiente atención por parte de los investigadores.

\section{El enfoque de beneficios compartidos en la literatura}

Fuera de América Latina, los investigadores han focalizado sus análisis en varios temas centrales de los BC: sus definiciones y modalidades operativas, la naturaleza del proceso de negociaciones implementados para su firma, los contenidos de los acuerdos, sus principales resultados y el debate acerca de la necesidad o no de contar con un marco legal específico que los regulen. Enseguida se describe cada caso.

\section{Definiciones y modalidades de beneficios compartidos}

El concepto de $\mathrm{BC}$ es de naturaleza multiescalar, ya que los mecanismos existentes pueden aludir a un nivel internacional, cuando dos o más países acuerdan cómo compartir los beneficios generados por un proyecto (frecuentemente hidroeléctrico) en una frontera común (Yu, 2008); a un nivel nacional, referido a cómo se distribuyen los beneficios al interior de un país, y a un nivel local, referente a cómo se comparten los beneficios con las comunidades locales afectadas por un proyecto extractivo (Peskett, 2011; Égré, 2007).

A nivel local, los BC corresponden a las negociaciones, firma de acuerdos y a su implementación entre una compañía extractiva sea pública o privada y una localidad que puede ser indígena o no indígena (Gross et al., 2005; Égré, 2007). En este artículo el objetivo es centrarse en los BC de nivel local que incluyen a comunidades indígenas.

Respecto a su definición, Woodward \& Company (2009: p. I-2) señalan que los $\mathrm{BC}$ son un "acuerdo escrito que es el resultado de un proceso de consulta acerca de un proyecto de extracción de recursos o de desarrollo propuesto, que

3 Recuperado el 5 de enero de 2015, de http://barricklatam.com/barrick/presencia/pascua-lama/comunidad/memorandum-de-entendimiento/2014-10-10/092526.html

G. Bustamante-Rivera, T. Martin | Beneficios compartidos y la gobernanza de la extracción de recursos naturales en territorios indígenas: aportes y limitaciones para Latinoamérica | Perfiles Latinoamericanos, 26(52) 
tiene el potencial de impactar los derechos o intereses indígenas de uno o más grupos indígenas en Canadá” (Traducción propia). ${ }^{4}$

No obstante, en términos conceptuales se deben separar los BC de las compensaciones, indemnizaciones y mitigaciones (Wang, 2012). Así, en rigor el $\mathrm{BC}$ consiste en los acuerdos exclusivamente en torno a las modalidades por las cuales las comunidades indígenas participan de los beneficios de un proyecto extractivo. Por ejemplo, para clarificar la distinción entre ambos enfoques, el mismo Wang señala que el dinero de las compensaciones se toma del presupuesto del proyecto, en cambio el BC proviene de sus ingresos. Sin embargo, en la mayoría de las experiencias registradas (en su modalidad de AIB) se negocian en conjunto compensaciones e indemnizaciones con beneficios, llegando a veces a confundirse.

De la experiencia canadiense destaca también la necesidad de esclarecer otras distinciones, a saber: entre BC y evaluación de impacto ambiental, y entre BC y aplicación del derecho a consulta indígena (Lukas-Amulung, 2009). En el primer caso, la dificultad consiste en clarificar su articulación, ya que en algunos territorios canadienses solo pueden iniciar la evaluación ambiental los proyectos que cuenten con un AIB ya firmado, mientras que en otros ambos procesos corren en forma separada. En el segundo, en ocasiones las negociaciones para la firma de un acuerdo de $\mathrm{BC}$ se consideran como aplicación del derecho indígena de ser consultados; sin embargo, esta relación entre derecho de consulta y BC no se ha precisado (Newman, 2014). Dado que en América Latina el derecho de consulta previa ha sido progresivamente establecido en los países que han ratificado el Convenio 169 de la Organización Internacional del Trabajo (en adelante, C169), la necesidad de precisar la relación entre negociaciones para firmar acuerdos de BC y aplicación del derecho a la consulta previa representan uno de los aspectos a considerar cuando se aplican mecanismos de BC. En los países que han ratificado el C169, el deber de consultar recae en el Estado y no en compañías privadas, por lo que las negociaciones emprendidas por compañías extractivas privadas pueden entenderse como delegación a terceras partes del deber de consulta.

Por otro lado, el significado que cobra el enfoque de BC varía según los actores involucrados: para las comunidades indígenas significa la posibilidad de beneficiarse de un proyecto en su territorio mediante la obtención de recursos para su desarrollo local (Wang, 2012; O'Faircheallaigh, 2010; Fidler \& Hitch, 2007; Prno, 2007), ganar empoderamiento como resultado de su

\footnotetext{
4 "a written agreement that is the outcome of a consultation process about a proposed resource extraction, project or development that has the potential to impact the Aboriginal rights or interests of one or more Aboriginal groups in Canada", dice el original en inglés.
} 
participación directa en las decisiones del proyecto (Perkett, 2011; Fidler \& Hitch, 2007) e incidir en la toma de decisiones en algo que les concierne (Fidler \& Hitch, 2007). Para los gobiernos significa disponer de un mecanismo para la inclusión social, la participación, la gobernabilidad, la justicia y la paz social (Wang, 2012). Y para las industrias extractivas significa la obtención de una "licencia social" que legitima sus proyectos, reduce los conflictos e incertidumbres (Peskett, 2011; Wang, 2012; Fidler \& Hitch, 2007; Fidler, 2010), materializa su responsabilidad social corporativa (Fidler \& Hitch, 2007) y les permite responder a la presión de la sociedad civil de que sus prácticas sean sustentables (Qureshy, 2006).

En lo que parece haber más consenso es en que las diversas modalidades de $\mathrm{BC}$ son el reconocimiento por parte de todos los actores de que la tradicional extracción de recursos naturales (sin participación significativa de las comunidades indígenas y sin que estas se beneficien de los proyectos en sus territorios) ya no es aceptable (Fidler \& Hitch, 2007; Hitch, 2006). Este sería el mayor aporte y la mejor promesa que ofrecen estos mecanismos para América Latina.

Respecto de las diversas modalidades de BC que existen, se cuenta con la clasificación de Égré (2007) y la del Ministerio de Recursos Naturales de Canadá ${ }^{\prime}$ que las distinguen según sus contenidos — desde acuerdos centrados en aspectos económicos a otros más integrales que incluyen una amplia gama de aspectos relevantes para la comunidad-y el grado de formalización de los acuerdos - desde protocolos de entendimiento de tipo general hasta los más específicos acuerdos de impactos de beneficios-. Égré (2007) diferencia entre los mecanismos monetarios o los no monetarios: los primeros consisten en que las comunidades reciben directamente de la industria extractiva una parte de los ingresos resultantes del proyecto, reciben financiamiento para programas de desarrollo para la comunidad, obtienen el traspaso total o parcial de la propiedad de la industria extractiva a la comunidad, reciben parte del cobro de impuestos que el gobierno obtiene por las operaciones del proyecto extractivo u obtienen tarifas rebajadas en el uso de la electricidad o de las aguas de riego. Los segundos se refieren al acceso de la comunidad a las aguas de regadío de una represa si se trata de un proyecto hidroeléctrico, al acceso de la comunidad a empleos relacionados con el proyecto extractivo y al uso de la infraestructura construida por el proyecto. En general, las experiencias concretas de BC utilizan más de una de estas modalidades.

En síntesis, los $\mathrm{BC}$ se definen como un componente de la gobernanza de los proyectos extractivos en territorios indígenas y no indígenas por cuyo medio

5 Recuperado el 16 de septiembre de 2013, de http://www.nrcan.gc.ca/sites/www.nrcan.gc.ca/files/mineralsmetals/files/pdf/abor-auto/aam-eac-e2013.pdf 
las comunidades afectadas participan del proceso de toma de decisiones para recibir directamente — además de indemnizaciones o compensaciones - parte de los beneficios que deriven del proyecto.

\section{Los beneficios compartidos como proceso}

La construcción de confianzas entre los actores implicados es un aspecto fundamental de los mecanismos de $\mathrm{BC}$, lo cual requiere que estas negociaciones se conciban como procesos a largo plazo que no terminan al firmar un acuerdo.

$\mathrm{Y}$ aunque el proceso y los contenidos de los acuerdos de BC son específicos a cada caso, el de la mina de Voisey Bay en la provincia de Labrador, Canadá, ejemplifica este tipo de negociaciones. De acuerdo a Laforce (2012b), el yacimiento de níquel de Voisey Bay se descubrió en el contexto de la reivindicación de los derechos de los cri y los inuit — que hasta entonces no estaban claramente delimitados - y de la regulación de las actividades de exploración minera en esa provincia. A mediados de los noventa, dicha minera (que desde 2006 pasó a ser propiedad de una compañía brasileña) comenzó las obras de infraestructura para dar lugar a la exploración. Ante esto las comunidades indígenas presentaron recursos judiciales para detener esas obras y poder realizar una evaluación ambiental adecuada. En 1997 se firmó un protocolo de acuerdo entre esas comunidades y los gobiernos federal y provincial, lo que permitió adoptar una evaluación de impacto ambiental basada en un modelo de desarrollo sostenible. Se incluyeron dimensiones sociales, económicas, culturales, recreacionales, espirituales, estéticas y biofísicas, más una mirada de diferenciación entre sexos. Además, se implementaron amplias consultas (con financiamiento del gobierno federal) que funcionaron durante tres ańos como una comisión medioambiental. Además el proyecto minero debió responder a 107 recomendaciones que incluyeron tres que para los indígenas eran estratégicos: $i)$ que las reivindicaciones territoriales indígenas fueran definidas antes del inicio del proyecto, ii) que las negociaciones tuvieran como resultado la firma de АIB con las comunidades afectadas, y iii) que se concluyeran acuerdos de cogestión medioambiental. En 2002 las organizaciones inuit e innu firmaron el AIB con la minera, incluyendo hacer negocios, empleos y la capacitación laboral de las comunidades involucradas. Se firmó de igual modo el Acuerdo de Gestión Ambiental de Voisey Bay que creó un comité de gestión ambiental compuesto de dos representantes por cada contraparte.

La negociación anterior contrasta con la de las centrales hidroeléctricas Pangue y Ralco de Endesa, S. A., construidas en tierras mapuches en el sur de Chile. Si bien en este caso hubo compensaciones individuales y la creación de un 
organismo promotor del desarrollo comunitario (la Fundación Pehuén), el proceso que fue altamente conflictivo al punto de requerir la intervención de la Corte Interamericana de Derechos Humanos y de llevar a la creación de la World Commission of Dams por parte del Banco Mundial. Este caso latinoamericano ilustra cómo las compensaciones individuales y la creación de fondos de desarrollo comunitario (ambas son formas de BC) por sí solos no aseguran resultados favorables para la comunidad y de legitimación ("licencia social") para la industria extractiva y el gobierno. El hecho confirma la importancia de generar relaciones de confianza a largo plazo y que en el contexto indígena ellas deben establecerse a nivel colectivo con los representantes de la comunidad, y no de forma individual.

O'Faircheallaigh (2009, 2010) y Weitzner (2006) destacan algunas de las serias limitaciones que pueden surgir durante la negociación entre comunidades y compañías mineras: la aparición de "burbujas de negociación" en las que el proceso se desvincula de otros más amplios que atañen a las comunidades (por ejemplo, de negociación de reivindicaciones territoriales más amplias con el gobierno, o de planes de desarrollo previos de la comunidad). Esto implicaría, en América Latina, prestar atención al contexto político más amplio en el que los pueblos indígenas buscan ocupar un mejor lugar en los Estados nación. Así, la participación de comunidades en negociaciones para firmar BC no reemplazará a otras más amplias y el logro de aquellos se entenderá en un marco que abarca reivindicaciones de los pueblos indígenas en cuestión.

Otro riesgo crítico en los $\mathrm{BC}$ es la inclusión de cláusulas de "confidencialidad" durante las negociaciones. Estas impiden a la comunidad y a la industria extractiva hacer públicos sus AIB, lo que limita la capacidad de la primera de crear alianzas con otros actores estratégicos a fin de posicionarse mejor ante la industria extractiva. Dichas cláusulas impiden que las comunidades aprendan de otras experiencias lo que les permitiría negociar en mejores condiciones y con mejor información acerca de la gama de $\mathrm{BC}$ posibles de obtener. Weitzner (2006) destaca la importancia de la dimensión temporal de las negociaciones toda vez que en la comunidad Lutsel K'e del pueblo dene sus ancianos no pudieron cumplir su responsabilidad tradicional de consejeros en la toma de decisiones comunitaria debido a que el gobierno impuso plazos excesivamente cortos para firmar el acuerdo final.

Otra limitación es que los AIB corresponden a negociaciones entre las comunidades indígenas y la industria extractiva, donde el gobierno no interviene. De ese modo, y como se trata de construir relaciones de confianza mutua de largo plazo son negociaciones que se dan en condiciones de profunda asimetría de poder entre industrias extractivas y comunidades (Hitch, 2006). Al respecto, y considerando los hallazgos de Arellano-Yanguas (2012) sobre la 
aplicación del $\mathrm{BC}$ en Perú, si en el ámbito anglosajón los actores implicados son comunidad indígena-industria extractiva-(Estado) (así, entre paréntesis, para resaltar su relativa ausencia), en Latinoamérica son comunidad indígenaindustria extractiva-(Estado)-sociedad civil, esta última conformada por ONG y movimientos sociales no indígenas que apoyan en las negociaciones a las que sí lo son.

Una última limitación es propia del caso minero y petrolero, la cual consiste en incluir el principio de libre entrada, esto es, que la actividad minera tiene prioridad por sobre cualquier otro uso del territorio. En este sentido, las negociaciones con la industria extractiva incorporan la certeza de que si el proyecto es viable entonces se contará con las autorizaciones gubernamentales para llevarlo a cabo y se realizará sin conflictos con las comunidades pues esto aumentaría los costos. En esta situación, el uso de BC permite que las industrias extractivas definan las compensaciones y beneficios que dará a la comunidad afectada.

\section{Los contenidos de los acuerdos de beneficios compartidos}

Fidler \& Hitch (2007) señalan que los contenidos de los AIB han evolucionado desde una primera etapa en que consistían en meros acuerdos socioeconómicos a los más recientes que son más integrales y contemplan mayor participación, transparencia y compromiso por parte de los firmantes.

Los principales contenidos de los acuerdos en general son los derechos e intereses sobre la tierra, la gestión ambiental, la protección de la herencia cultural, los beneficios financieros, de empleo y de capacitación, el desarrollo de negocios y el consentimiento y apoyo indígena al proyecto (O'Faircheallaigh, 2003).

En la estructura característica de estos acuerdos, Shanks (2006) identifica varios tipos de cláusulas: introductorias, de fomento de empleo y capacitación, de operaciones de negocios de las comunidades, de apoyo de la industria a aspectos sociales, culturales y comunitarios para mitigar los impactos negativos, de compensaciones y de beneficios financieros, de protección del medioambiente y la cultura, y las referentes a aspectos procedimentales.

A modo de balance, cuando no hay un marco legal que regule estos acuerdos, los contenidos posibles son prácticamente ilimitados y dependen de lo que se pacte entre la comunidad y la industria extractiva, y en ocasiones con el gobierno. Lo que no sucede en presencia de marcos legales reguladores.

Una revisión de la escasa literatura para América Latina muestra que se han usado beneficios del tipo fondos de desarrollo comunitario (Ralco, Chile, a través de la Fundación Pehuén; la mina Marlin, en Guatemala), acceso prioritario 
a empleo (San Isidro, Bolivia), y planes de etnodesarrollo (Urrá I, Colombia). Sin embargo, en todos estos casos la idea del BC es más bien secundaria y lo que predomina son las indemnizaciones desproporcionadamente bajas en relación con los daños y que se definen muy tardíamente ( Itaipú, entre Brasil y Paraguay; Ralco, en Chile).

\section{Resultados del beneficio compartido en comunidades indígenas}

Paradojalmente y pese a su importancia no hay muchos estudios sobre los resultados reales de los $\mathrm{BC}$ en las comunidades. Entre los más críticos se encuentra el trabajo de O'Faircheallaigh \& Corbett (2005) en el que los autores analizan 45 acuerdos firmados en distintas regiones de Australia concluyendo que no necesariamente facilitan la participación indígena en la gestión ambiental e incluso a veces reducen su participación. A similares conclusiones llegan Fidler \& Hitch (2007) quienes observan que los AIB en Canadá no benefician a las comunidades en el largo plazo, e incluso pueden impactar negativamente el medio ambiente de su entorno (Hitch, 2006).

En un estudio sobre la percepción respecto de los AIB firmados entre comunidades indígenas canadienses y compañías mineras, Prno (2007) indica que, en general, las primeras advierten que tienen mejores resultados cuando existen esos acuerdos. No obstante, también concluye que los beneficios económicos directos llegan solo a aquellos que obtienen empleos en las minas y no a toda la comunidad, que la construcción de confianzas que se produce es más bien limitada, que la comunidad analiza los beneficios en forma global sin separar los distintos AIB existentes, y que lo más importante para las comunidades indígenas es que los acuerdos sirven para el reconocimiento de sus derechos.

En uno de los pocos estudios que intenta recoger la perspectiva de las comunidades, Weitzner (2006) describe la insatisfacción de estas ante el producto de los acuerdos debido a los malentendidos iniciales sobre su amplitud: no habían considerado en las negociaciones sus derechos establecidos en su tratado con la Corona Británica; no se habían definido claramente las comunidades beneficiadas; habían aceptado una agencia independiente de monitoreo ambiental meramente consultiva que no fue tomada en cuenta por la industria extractiva; y habían surgido tensiones dentro de las comunidades y entre las comunidades durante las negociaciones y al distribuir los beneficios.

Se han identificado diversos factores en la base de los resultados negativos de estos acuerdos, tales como los problemas en sus contenidos, de las capacidades de las comunidades, de las industrias extractivas o de las características del proyecto (Dreyer \& Myers, 2004). Sin embargo, el factor más relevante de todos

G. Bustamante-Rivera, T. Martin | Beneficios compartidos y la gobernanza de la extracción de recursos naturales en territorios indígenas: aportes y limitaciones para Latinoamérica | Perfiles Latinoamericanos, 26(52) 
es el que destaca Hitch (2006) para el caso canadiense: el desigual poder entre comunidades e industrias extractivas que negocian las modalidades de BC sin intermediarios y con un Estado que se abstiene de intervenir o que actúa en colusión con las industrias extractivas.

En cuanto a los factores de éxito del BC, Gibson (2006) identifica como principales motivos para Voisey Bay en Canadá el uso de evaluaciones de impacto centrados en la sustentabilidad, el uso de múltiples iniciativas complementarias (donde los AIB eran una entre muchas iniciativas), la redistribución de poder entre las partes y el uso de la deliberación y de la evaluación permanente.

\section{¿Necesita el beneficio compartido un marco legal?}

El debate de si el BC necesita o no un marco legal se debe a que gran parte de las experiencias han tenido lugar en Canadá y en específico bajo la modalidad de los AIв, lo que ha teñido el análisis internacional del tema.

En Canadá los AIB corresponden a acuerdos entre comunidades indígenas e industrias extractivas (generalmente mineras) que, debido a que se producen fuera de un marco legal específico, son considerados como contratos entre privados. Su relación con las evaluaciones ambientales es menos consensual: para O'Faircheallaigh (2010) hay una relación negativa entre ambas; para Fidler (2010) y Prno (2007), una y otra se complementan si comienzan en etapas tempranas del proyecto extractivo; en tanto que, para Knotsch et al. (2010) y Fidler \& Hitch (2007), estos acuerdos son parte del marco legal de las evaluaciones ambientales. Así, comunidades indígenas e industrias extractivas inician negociaciones y firman sin estar legalmente obligados a hacerlo, pero una vez firmados los acuerdos se vuelven vinculantes y ante ellos el gobierno asume una postura de no intervención. Al ser acuerdos entre privados las industrias extractivas pueden imponer las cuestionadas cláusulas de confidencialidad (O'Faircheallaigh, 2010).

Lo anterior es así porque la práctica de los acuerdos de BC comenzó cuando en Canadá no existía un marco legal relativo al derecho de consulta de las comunidades indígenas, el cual estableció la Corte Suprema apenas en 2004 (Affaires Indiennes et du Nord Canada, 2011). De esta forma, en Canadá un régimen de negociaciones de $\mathrm{BC}$ se da sin regulación legal y en un contexto político que Laforce (2012a) denomina de "ausencia selectiva" del Estado. El Estado "elige" no intervenir pese a que tiene el deber constitucional de consultar a las comunidades indígenas, favoreciendo de este modo a las industrias extractivas, mismas que pueden imponer los términos de las negociaciones y 
legitimar su operación. Es un favorecimiento que se fundamenta en el principio de libre entrada ya señalado.

En los países latinoamericanos que han ratificado el C169 — que son mayoría- las negociaciones entre la industria extractiva y las comunidades indígenas se deberían entender bajo ese marco legal, el cual reconoce tanto el derecho de consulta previa como el de las comunidades de beneficiarse de los proyectos que operan en sus territorios. ${ }^{6}$ Así, en los países latinoamericanos signatarios del C169 las negociaciones para acordar BC podrían concebirse como formas de delegación de aspectos procedimentales de la consulta previa, pero donde la responsabilidad final recae siempre en el Estado, el cual debe regular las negociaciones a fin de reducir las asimetrías de poder.

Como las consultas previas buscan el CPLI de las comunidades indígenas, los contenidos incluidos en los acuerdos facilitarían que ellas den su consentimiento después de evaluar que los efectos negativos no fueran significativos y que estos se encuentren adecuadamente compensados por los BC, además de que puedan obtener beneficios suplementarios. De ser así, los eventuales BC serían parte de los cálculos que las comunidades llevan a cabo para definir sus estrategias y objetivos frente a las consultas respecto a proyectos extractivos en su territorio. Según Arellano-Yanguas (2012), la negociación de beneficios directos da lugar a que las comunidades incrementen el conflicto a modo de estrategia para alcanzar mejores beneficios. Sin negar esta posibilidad, se debe decir que los BC facilitan el diálogo y el CPLI de las comunidades que evalúan que los potenciales daños o beneficios de un proyecto. Sin embargo, también se reconoce que la extrema pobreza de las comunidades indígenas puede forzarlos a aceptar $\mathrm{BC}$ - aun cuando el proyecto represente amenazas serias a sus culturas - o a dividirse entre los que aceptan negociar y los que lo rechazan.

De todas formas, quedaría por resolver la articulación de la práctica de los $\mathrm{BC}$ con los procedimientos de consulta definidos legalmente y con los mecanismos de evaluación ambiental de cada país latinoamericano. En este sentido, el escenario más favorable para las comunidades indígenas sería definir la firma de acuerdos de $\mathrm{BC}$ como una condición obligatoria previa antes de pasar a la etapa de evaluación ambiental.

6 La Declaración de la Naciones Unidas sobre los Derechos de los Pueblos Indígenas (DNUDPI) establece que los pueblos indígenas tienen derecho a una indemnización o reparación "justa", "equitable" y "correcta" en casos de desplazamientos o reinstalación (artículos 10, 28 y 32), así como de mitigación cuando hay impacto negativo a nivel ambiental, económico, social, cultural o espiritual (artículo 32). Por su parte, el C169 de la orT determina que ante explotación de recursos "los pueblos interesados deben, cada vez que sea posible, participar de los beneficios resultantes de tales actividades y deben recibir una indemnización equitativa ante todo daño que pudieran sufrir por tales actividades” (artículo 15).

G. Bustamante-Rivera, T. Martin | Beneficios compartidos y la gobernanza de la extracción de recursos naturales en territorios indígenas: aportes y limitaciones para Latinoamérica | Perfiles Latinoamericanos, 26(52) 


\section{Conclusiones}

Los $\mathrm{BC}$ son un mecanismo de gobernanza de la extracción de recursos naturales en territorios indígenas que se han aplicado intensivamente en la minería e hidroelectricidad en el mundo anglosajón y en menor medida en hidroelectricidad en otras regiones del mundo, los cuales han sido promovidos por el Banco Mundial y por algunas industrias extractivas.

En América Latina, los BC se han utilizado en forma más limitada con resultados frecuentemente adversos además de que se les ha estudiado muy poco. Mientras que la importancia que los gobiernos de la región le dan dado al desarrollo basado en la extracción de recursos naturales en conjunto con el predominio de conflictos y efectos negativos en las comunidades ante las formas de gobernanza impulsadas y que sean mineras canadienses las que han protagonizado la mayor parte de las experiencias de $\mathrm{BC}$ en la zona, llevan a pensar que estos mecanismos habrán de consolidar su presencia en Latinoamérica, lo que hace necesario el análisis de sus aportes, limitaciones y condiciones de aplicación.

De acuerdo a la literatura, uno de los aportes de los BC es que con su innovación en la gobernanza muestran que los mecanismos en los que las comunidades indígenas no participan ni se benefician de los proyectos que operan en sus territorios ya no son aceptables. En tanto que a los gobiernos les aportan legitimidad en la gobernanza de estas actividades, a las industrias extractivas una licencia social que les reduce la incertidumbre de sus inversiones, costos de litigios judiciales y les mantienen una imagen corporativa positiva. Para las comunidades indígenas los aportes son menores, ya que aunque les abre un espacio de participación y de cierta influencia sobre los proyectos, su real aporte económico y social es más bien difuso. Aun así, los BC conducen a un esquema de ganador/ganador para industria extractiva y comunidad.

Los $\mathrm{BC}$ a la vez representan limitaciones y riesgos para las comunidades indígenas. La principal limitación es la asimetría de poder entre industrias extractivas y comunidades indígenas, lo que se acentúa cuando se da la "ausencia selectiva” del Estado (Szablowski, 2007. Citado en Laforce, 2012a). En rigor, en las negociaciones de $\mathrm{BC}$ no se discute si el proyecto se va a realizar o no, sino estrictamente las compensaciones, las medidas de mitigación y las formas de participación de las comunidades de los beneficios que de él se deriven. Otras limitaciones son las cláusulas de confidencialidad y las "burbujas de negociación”, pues obligan a que las comunidades apoyen el proyecto y se abstengan de emprender medidas judiciales contra él, lo que abre la posibilidad de que la industria extractiva imponga los contenidos de los acuerdos.

La revisión de la literatura especializada sugiere que los BC conllevan diversos riesgos para las comunidades indígenas en América Latina. Por un lado, el 
que sean usados en condiciones de "ausencia selectiva" del Estado o de "broker state" (Urteaga-Crovetto, 2012) o de que las industrias extractivas coopten al Estado y se produzcan incluso mayores asimetrías de poder ya que entonces este último decide favorecerlas por encima de los intereses de las comunidades. Un riesgo más es que ante la debilidad generalizada en la aplicación sustantiva del C169 (Bustamante, 2015), los BC reemplacen las consultas previas o que se consideren como expresión de CPLI, a pesar de que la relación entre BC y CPLI no es clara todavía y que estas negociaciones son principalmente entre industrias extractivas privadas y comunidades indígenas. Pero el riesgo central consiste en que ante la debilidad estructural de muchos Estados de la región, con los acuerdos de $\mathrm{BC}$, las industrias extractivas asuman roles propios del Estado — por ejemplo, financiando la salud o la educación-, lo que aumentaría la asimetría de poder entre comunidad e industria. Y cuando la participación indígena se restringe a las negociaciones de $\mathrm{BC}$, las comunidades corren el riesgo de aceptar proyectos que social, cultural y medioambientalmente son inaceptables. Debe añadirse que, por las condiciones de pobreza de las comunidades indígenas en América Latina, la "promesa" de obtener recursos materiales o económicos con los proyectos extractivos les origina tensiones, divisiones internas y el consentimiento de proyectos que deterioran su medioambiente y vida social y cultural. Un ulterior riesgo es que la industria extractiva imponga negociaciones individuales con cada miembro o familia de la comunidad y no con la autoridad representativa, lo que lesiona la organización social comunitaria y vulnera el principio de participación comunitaria como derecho colectivo.

Finalmente, la participación de las comunidades indígenas de los ingresos por concepto de las operaciones de los proyectos extractivos en o cerca de los territorios indígenas ya la mandatan el C169 y la Declaración de la Naciones Unidas sobre los Derechos de los Pueblos Indígenas. Acerca de esto, la literatura especializada muestra que en América Latina la negociación de BC tendrá legitimidad solo si cumple varias condiciones esenciales: $i$ ) que la negociación e implementación de los $\mathrm{BC}$ estén claramente articuladas a la aplicación sustantiva del C169, otorguen un rol activo a los Estados, y concuerden con los planes endógenos de desarrollo de las propias comunidades; ii) que los Estados sean activos regulando la aplicación de los $\mathrm{BC}$ e impidiendo la imposición de cláusulas de confidencialidad durante la negociación y para el público general tras la firma del acuerdo; iii) que los Estados impidan la imposición de cláusulas que restrinjan que la comunidad ejerza sus derechos como el de interponer medidas judiciales contra la industria extractiva; y iv) que las industrias extractivas consulten desde un inicio a las autoridades representativas de las comunidades, respetando estrictamente sus protocolos y dialogando con sus miembros solo 
bajo ese esquema, sancionándose toda negociación que produzca la división en las comunidades.

En un escenario de articulación con la aplicación del C169, en condiciones ideales los $\mathrm{BC}$ solo deberían utilizarse en proyectos que cuenten con el CPLI de la comunidad por lo que no tendrían que negociarse compensaciones y beneficios como mecanismo que los legitime. Una forma de aplicar los $\mathrm{BC}$ en mejores condiciones de negociación para las comunidades indígenas es cuando negociación y firma se asumen como requisitos previos para el ingreso y aprobación de un proyecto en la evaluación ambiental.

Por último, debe señalarse que los $\mathrm{BC}$ acordados no deben reemplazar el papel del Estado en la provisión de educación, salud e infraestructura, ni este último debe condicionar el financiamiento de estos servicios en función de los acuerdos de BC firmados por la comunidad.

\section{Referencias}

Affaires Indiennes et du Nord Canada. (2011). Consultation et accommodement des Autochtones: lignes directrices actualisées à l'intention des fonctionnaires fédéraux pour respecter l'obligation de consulter. Ottawa: Ministère des Affaires Indiennes et du Nord Canada.

Anaya, J. (2011). Report of the Special Rapporteur on the Rights of Indigenous Peoples: Extractive Industries Operating within or near Indigenous Territories. Ginebra: Human Rights Council, United Nations.

Arellano-Yanguas, J. (2012). Mining and conflict in Peru: Sowing the minerals, reaping a hail of stones. En Bebbington, A. (Ed.). Social Conflict, Economic Development and Extractive Industry: Evidence from South America (pp. 89-111). Nueva York, Routledge.

Auty, R. (1998). Resource Abondance and Economic Development: Improving the Perfomance of Resource-rich Countries. Helsinsky: onU World Institute for Development Economics Research.

Bebbington, A. (2012). Extractive industries, socio-environmental conflicts and political economy transformations in Andean America. En Bebbington, A. (Ed.). Social Conflict, Economic Development and Extractive Industry: Evidence from South America (pp. 3-26). Nueva York: Routledge.

Bebbington, D. (2012). State-Indigenous tensions over hydrocarbon expansión in the Bolivian Chaco. En Bebbington, A. (Ed.). Social Conflict, Economic Development and Extractive Industry: Evidence from South America (pp. 134-152). Nueva York: Routledge. 
Boschini, A., Petterson, J. \& Roine, J. (2007). Resource Curse or Not: A Question of Appropriability. The Scandinavian Journal of Economics, 109(3), 593-617.

Bustamante, G. (2015, marzo). The Right to Consultation and Free, Prior and Informed Consent in Latin America: The Governmentality of the Extraction of Natural Resources. Revue québécoise de droit international, 179-197.

Campbell, B. \& Laforce, M. (2010). La réforme des cadres réglementaires dans le secteur minier : les expériences canadienne et africaine mises en perspective. Recherche amérindiennes au Québec, XL(3), 69-84.

Campbell, B. \& Sarrasin, B. (2012). Introduction. En Laforce, M., Campbell, B. \& Sarrasin, B. (Eds.). Pouvoir et régulation dans le secteur minier: leçons à partir de l'expérience canadienne (pp. 1-8-). Québec: Presses de l’Université du Québec.

Casalis, A. \& Trinelli, A. (2013). El desarrollo territorial en Argentina: oportunidades y desafíos de la explotación de los recursos minero (2002-2012). Revista Estado y Políticas Públicas, (1), $97-114$.

Dietz, S., Neumayer, E. \& De Soysa, I. (2007). Corruption, the Resource Curse and Genuine Saivings. Environment and Development Economics, 12(1), 33-53.

Dreyer, D. \& Myers, Heather, (2004). Impact and Benefit Agreements: Do the Ross River Dena Benefit from Mineral Projects? Informe Final presentado ante el Northern Land Use Institute, University of British Columbia, Prince George, Canadá.

Dunning, T. (2005). Resource Dependence, Economic Perfomance, and Political Stability. Journal of Conflict Resolution, 49(4), 451-482.

Égré, D. (2007). Compendium on Relevant Practices 2nd Stage: Revised Final Report Benefit Sharing Issue: Dams and Development Project. Montreal: United Nations Programme.

Fidler, C. (2010). Increasing the Sustainability of a Resource Development: Aboriginal Engagement and Negociated Agreements. Environmental Development Sustaintability, (12), 233-244.

Fidler, C. \& Hitch, M. (2007). Impact and Benefit Agreements: A Contentious Issue for Environmental and Aboriginal Justice. Environments Journal, 35(2), 49-69.

Gibson, R. (2006). Sustainability assessment and conflict resolution: Reaching agreement to proceed with the Voisey's Bay nickel mine. Journal of Cleaner Production, 14(3-4), 334-348.

Gudynas, E. (2012). Estado compensador y nuevos extractivismos : las ambivalencias del extractivismo sudamericano. Nueva Sociedad, (237), 128-146.

G. Bustamante-Rivera, T. Martin | Beneficios compartidos y la gobernanza de la extracción de recursos naturales en territorios indígenas: aportes y limitaciones para Latinoamérica | Perfiles Latinoamericanos, 26(52) 
Hitch, M. (2006). Impact and Benefit Agreements and the political ecology of mineral development in Nunavut. Tesis para obtener el grado de Doctor en Filosofía en Geografía, Universidad de Waterloo. Waterloo, Ontario, Canadá.

Johnston, B. \& García-Downing, C. (2004). Hydroelectric Development on the Bío-Bío River, Chile: Anthropology and Human Rights Advocacy. En Blaser, M., Feit, H. A. \& McRae, G. (Eds.). In the Way of Development: Indigenous Peoples, Life Projects and Globalization (pp. 211-234). Londres/Nueva York: Zed Books.

Knotsch, C., Siebenmorgen, P. \& Bradshaw, B. (2010). Les "Ententes sur les répercussions et les avantages": des occassions ratées? Recherches amérindiennes au Québec, 40(3), 59-68.

Laforce, M. (2012a). Processus de dialogue et ententes négociées: réponses durables aux conflits? En Laforce, M., Campbell, B. \& Sarrasin, B. (Ed.). Pouvoir et régulation dasn le secteur minier: Leçons à partir de l'expérience canadienne (pp. 1919-251). Québec: Presses de l'Université du Québec.

Laforce, M. (2012b). Régulation du projet minier de Voisey’s Bay au Labrador: Vers un rééquilibrage des pouvoirs dans certains contextes politiques et institutionnels? En Laforce, M., Campbell, B. \& Sarrasin, B. (Ed.). Pouvoir et régulation dasn le secteur minier: Leçons à partir de l'expérience canadienne (pp. 157-189). Québec: Presses de l'Université du Québec.

Lukas-Amulung, S. (2009). The Rules of Engagement? Negotiated Agreements and Environmental Assessment in the Northwest Territories, Canada. Memoria para obtener el grado de Master of Arts in Environment and Management. Victoria, Canadá: Royal Roads University.

Newman, D. (2014). Revisiting the Duty to Consult Aboriginal Peoples. Saskatoon, Canadá: Purich Publishing.

O’Faircheallaigh, C. (2010). Aboriginal Mining Company Contractual Agreements in Australie and Canada: Implications for Political Autonomy and Community Development. Canadian Journal of Development Studies, 30(1-2), 69-86.

O’Faircheallaigh, C. (2009). Corporate-Aboriginal Agreements on Mineral Development: The Wider Implications of Contractual Arrangements. Ponencia presentada en la Conferencia Repensando las Industrias Extractivas realizada del 5 al 7 de marzo 2009, York University, Toronto, Canadá.

O’Faircheallaigh, C. (2003). Implemening Agreements Between Indigenous Peoples and Resource Developers in Australia and Canada. Aboriginal Politics and Public Sector. Management Research Paper, núm. 3. Griffith University. 
O'Faircheallaigh, C. \& Corbett, T. (2005). Indigenous Participation in Environmental Management of Mining Projects: The Role of Negociated Agreements. Environmental Politics, 14(5), 629-647.

Ostau, F. \& Nińo, L. (2012). La responsabilidad social empresarial en las empresas del sector carbonífero colombiano: lecciones y experiencias de las transnacionales mineras en el marco de los estándares internacionales determinados por la Organización Internacional del Trabajo. Diálogo de Saberes, (36), 81-98.

Pendergast, S., Clarke, J. \& Van Kooten, C. (2011). Corruption, Development and the Curse of Natural Resources. Canadian Journal of Political Sciences/Revue canadienne de sciences politiques, 44(2), 411-437.

Prno, J. (2007). Assessing the Effectiveness of Impact and Benefit Agreements from the Perspective of their Aboriginal Signatories. Memoria para la obtención del grado de Master of Arts. Guelph: Universtity of Guelph.

Qureshy, S. (2006). Landlords and Political Traps: How Mineral Exploration Companies Seek Acces to First Nation Territory. Memoria para la obtención del grado de Master of Arts. Ottawa: Carleton University.

Reed, D. (2002). Resource extraction industries in developing countries. Journal of Business Ethics, 39(3), 199-226.

Riva, P. de la. (2010). Proceso de consulta y participación proyecto de exploración sísmica 2D campos Tacobo y Tajibo, Bloque San Isidro. En Bascopé Sanjinés, I. (Coord.). Lecciones aprendidas sobre consulta previa (pp. 159-232). La Paz: CEJIS.

Ross, M. (1999). The Political Economy of the Resource Curse. World Politics, (51), 297-322.

Salant, S. (1995). The Economics of Natural Resource Extraction: A Primer for Development Economists. The World Bank Research Observer, 10(1), 93-111.

Sawyer, S. \& Gomez, E. (2012). Transnational governmentality in the context of resource extraction. En Sawyer, S. \& Gomez, E. (Eds.). The politics of resource extraction: indigenous peoples, multinational corporations and the State (pp. 1-8). Nueva York: United Nations Research Institute for Social Development/Palgrave MacMillan.

Shanks, G. (2006). Sharing in the Benefits of Resource Developments: A Study of the First NationsIndustry Impacts Benefits Agreements. Ottawa: Public Policy Forum.

Urteaga-Crovetto, P. (2012). The Broker State and the Inevitability of Progress: The Camisea Project and Indigenous Peoples in Peru. En Sawyer, S. \& Gomez, E. (Eds.). The politics 
of resource extraction: indigenous peoples, multinational corporations and the State (pp. 103128). Nueva York: United Nations Research Institute for Social Development/Palgrave MacMillan.

Wang, C. (2012, junio). A guide for Local Benefit Sharing in Hydropower Projects. Social Development Working Papers of the World Bank, (128).

Weinthal, E. \& Jones, P. (2006). Combating the Resource Curse: An Alternative Solution to Managing Mineral Wealth. Perspectives in Politics, 4(1), 35-53.

Weitzner, Vi. (2006). "Dealling full force": Lutsel K'e Dene First Nation's Experience Negotiating with Mining Companies. Ottawa: The North-South Institute.

Woodward \& Company. (2009). Benefit Sharing Agreements in British Columbia: a Guide for First Nations, Businesses and Governments. Informe preparado para Ecosystems-Based Management Working Group, Victoria, Canadá.

World Commission on Dams. (2000). Dams and Development: A new Framework for DecisionMaking. Londres: World Commission on Dams.

Yu, W. (2008). Benefit Sharing in International Rivers: Findings from the Senegal River Basin, the Columbia River Basin, and the Lesotho Highlands Water Project. Washington, D.C.: World Bank.

Zarsky, L. \& Stanley, L. (2011). Buscando oro en el altiplano de Guatemala: Beneficios económicos y riesgos ambientales de la Mina Marlin. Medford, Massachusetts: Instituto de Desarrollo Global y Medio Ambiente. 